\title{
Natural course of autoimmune thyroiditis in type 1 diabetes: association with gender, age, diabetes duration, and puberty
}

\author{
O Kordonouri, R Hartmann, D Deiss, M Wilms, A Grüters-Kieslich
}

Arch Dis Child 2005;90:41 1-414. doi: 10.1136/adc.2004.056424

\author{
See end of article for \\ authors' affiliations

..................... \\ Correspondence to: \\ Dr O Kordonouri, Klinik für \\ Allgemeine Pädiatrie, \\ Otto-Heubner-Centrum, \\ Charité Universitätsmedizin \\ Berlin, Campus Virchow- \\ Klinikum, Augustenburger \\ Platz 1, 13353 Berlin, \\ Germany; \\ olga.kordonouri@ \\ charite.de \\ Accepted \\ 23 September 2004
}

\begin{abstract}
Aims: To investigate the natural history and incidence of autoimmune thyroiditis (AIT) in paediatric patients with type 1 diabetes (TID).

Methods: Since 1990, annual screening for thyroid disease has been performed in children and adolescents with TID. Antibodies against thyroperoxidase (anti-TPO) and thyroglobulin (anti-TG) as well as TSH were measured in 659 patients (54.3\% boys). In 126 patients, anti-TPO and anti-TG levels were followed at yearly intervals from onset up to five years of TID. Anti-TPO above $30 \mathrm{U} / \mathrm{ml}$ and anti-TG above $20 \mathrm{U} / \mathrm{ml}$ were considered positive, values above $100 \mathrm{U} / \mathrm{ml}$ as significantly raised and indicative of AIT. L-thyroxine treatment was started if TSH was higher than $4.5 \mu \mathrm{U} / \mathrm{ml}$ and/or thyroid gland enlargement on thyroid ultrasound was present.

Results: At initial screening, $15.4 \%$ of patients had raised anti-TPO and $14.4 \%$ anti-TG. Girls had more frequently raised antibodies than boys. Sixty two patients $(9.4 \%, 61 \%$ girls) required treatment with L-thyroxine. The cumulative incidence (SE) of AIT after 10 years of diabetes was 0.14 (0.02), being significantly higher in females $(0.18(0.03))$, particularly after the age of 12 years. At TID onset, positive anti-TPO and anti-TG were present in 21 of 126 patients (16.7\%), each. All patients with significantly increased values of anti-TPO $(\mathrm{n}=17,148-5340 \mathrm{U} / \mathrm{ml})$ and anti-TG $(\mathrm{n}=11,140-2000 \mathrm{U} / \mathrm{ml})$ at T1D onset remained positive during the following five years.

Conclusions: For early detection of autoimmune thyroiditis in children with TID, measurement of anti-TPO and TSH at TID onset and in yearly intervals after the age of 12 years is recommended.
\end{abstract}

T he prevalence of autoimmune thyroiditis is reported to be significantly higher among young patients with type 1 diabetes than in the age matched general population. ${ }^{12}$ Chronic autoimmune thyroiditis is characterised by the presence of thyroid specific autoantibodies in serum and by varying degrees of thyroid dysfunction. ${ }^{3}$ Moreover, ultrasound studies of the thyroid gland have shown that as well as gland enlargement, typical patterns of parenchymal hypoechogenicity are present in patients with autoimmune thyroiditis. ${ }^{4}$ However, up to now longitudinal studies assessing the risk of autoimmune thyroiditis are lacking and there is no consensus on screening for autoimmune thyroiditis in patients with type 1 diabetes.

The International Society for Pediatric and Adolescent Diabetes (ISPAD) Consensus Clinical Guidelines 2000 suggest that thyroid function and thyroid antibody tests should be considered close to the time of diagnosis and repeated if clinical circumstances suggest the possibility of thyroid disease. ${ }^{5}$ The guidelines make no statement on screening of asymptomatic patients. The lack of large prospective studies on epidemiology and development of autoimmune thyroiditis in young patients with type 1 diabetes makes difficult the establishment of unique diagnostic procedures in this collective. Moreover, the estimation of cumulative incidence and relative risk for the development of autoimmune thyroiditis can only be based on longitudinal observation data by means of regular examination.

The aim of this study was to examine the incidence and the course of autoimmune thyroiditis in young patients with type 1 diabetes using a long term regular screening programme for thyroid disease. Particular interest was focused on the development of thyroid disorders (hypothyroidism, subclinical hypothyroidism, goitre) requiring treatment with L-thyroxine and the investigation of clinical parameters influencing progression of AIT. Furthermore, the role of thyroid specific autoantibodies as a screening tool was analysed and evaluated.

\section{PATIENTS AND METHODS}

In 1990, a longitudinal screening of thyroid function was commenced at the Charité Children's Hospital of the Humboldt University of Berlin, Germany, in order to detect early thyroid disorders in young patients with type 1 diabetes. Thyroid function tests $\left(\mathrm{T}_{4}, \mathrm{~T}_{3}\right.$, and TSH) were performed by routine assays during the annual examinations, while antibodies against thyroperoxidase (anti-TPO) and thyroglobulin (anti-TG) were measured subsequently, if abnormalities of thyroid hormones and, particularly TSH increase, were present. Since 1996, anti-TPO and anti-TG measurement was added to the primary screening programme. In the presence of abnormal thyroid function tests and/or antibodies, ultrasound studies of the thyroid gland were performed. Treatment with L-thyroxine $\left(100 \mu \mathrm{g} / \mathrm{m}^{2}\right.$ body surface area) was started, if TSH increase $(\geqslant 4.5 \mu \mathrm{U} / \mathrm{ml})$ on two subsequent measurements and/or thyroid gland enlargement (thyroid gland volume $>97$ th age related centile ${ }^{6}$ ) with diffuse parenchymal hypoechogenicity in ultrasound examination $^{4}$ were present. The TSH normal range in healthy children of the iodine replete area of Berlin was estimated to be $0.3-3.8 \mu \mathrm{U} / \mathrm{ml}$; TSH values above $4.0 \mu \mathrm{U} / \mathrm{ml}$ were regarded as suspicious for an impaired thyroid function at the Charité Medical Centre.

Abbreviations: AlT, autoimmune thyroiditis; $\mathrm{CV}$, coefficient of variation; $\mathrm{SE}$, standard error; TID, type 1 diabetes; TG, thyroglobulin; TPO, thyroperoxidase; TSH, thyroid stimulating hormone 
From January 1990 to December 2003, 659 patients (358 boys, 301 girls) with type 1 diabetes underwent regular screening for thyroid dysfunction. Their median age at initial screening examination was 10.9 years (range 0.1-24 years), and their diabetes duration 1.2 years ( $0-14.8$ years). A further patient ( 16 year old girl) with Down's syndrome, coeliac disease, and clinical signs of hyperthyroidism was diagnosed to have Graves' disease. This patient was excluded from the study.

In a subgroup of 126 children and adolescents ( 80 boys, 46 girls), anti-TPO and anti-TG levels were followed at yearly intervals from onset of diabetes up to five years (median age at type 1 diabetes onset 9.0 years, range $0.1-15$ years) in order to evaluate antibody fluctuations during that period.

Antibodies to thyroglobulin (anti-TG) were determined by radioimmunoassay (DYNOtest anti-TG ${ }_{n}$, BRAHMS Diagnostica, Berlin, Germany); intra- and inter-assay coefficients of variation $(\mathrm{CV})$ were $7.5 \%$ and $5.5 \%$, respectively. The detection limit was $5.5 \mathrm{U} / \mathrm{ml}$, and the analytical assay sensitivity $20 \mathrm{U} / \mathrm{ml}$. A titre exceeding $20 \mathrm{U} / \mathrm{ml}$ was considered positive, and above $100 \mathrm{U} / \mathrm{ml}$ was considered significantly increased. Anti-TG measurements were available in 637 of 659 patients $(96.7 \%)$.

Antibodies to thyroperoxidase (anti-TPO) were determined radioimmunometrically (DYNOtest anti-TPO $\mathrm{n}_{\mathrm{n}}$ BRAHMS Diagnostica); intra- and inter-assay CV were $4.3 \%$ and $9.1 \%$, respectively. The detection limit was $5.5 \mathrm{U} / \mathrm{ml}$ and the analytical assay sensitivity $30 \mathrm{U} / \mathrm{ml}$. A titre exceeding $30 \mathrm{U} /$ $\mathrm{ml}$ was considered positive, above $100 \mathrm{U} / \mathrm{ml}$ as significantly increased. Anti-TPO measurements were available in 637 of 659 patients $(96.7 \%)$. The prevalence of anti-TPO antibodies in healthy children in Berlin has been found to be 3.4\%. ${ }^{7}$

\section{Statistical analysis}

Data were analysed using the Statistical Package for Social Sciences (SPSS 11.0.1). Group differences for continuous variables were assessed using the Mann-Whitney U-test. Differences in frequencies for categorical variables were tested by the $\chi^{2}$ test. Data are presented as median (range). As a measure of the probability to develop an autoimmune thyroiditis after diabetes onset, cumulative incidence was used and calculated using the Kaplan-Meier analysis; results are given as mean (SE). Significant differences were assumed for $\mathrm{p}<0.05$.

\section{RESULTS}

In the total group, 98 of 637 patients (15.4\%) had increased anti-TPO antibodies and 92 of 637 patients (14.4\%) were positive for anti-TG at the start of screening. Girls had more frequently increased anti-TPO antibodies than boys (58 of $291(19.9 \%) v 40$ of $346(11.6 \%), p=0.004)$ as well as increased anti-TG antibodies (54 of $291(18.6 \%) v 38$ of 346 $(11.0 \%), p=0.007)$.

During the study period, 62 of 659 patients $(9.4 \%)$ required treatment with L-thyroxine and another 40 patients $(6.1 \%)$ had thyroid positive antibodies, but did not meet the criteria for L-thyroxine substitution after a median diabetes duration of 3.9 years (0.2-12.4 years). Furthermore, 26 antibody positive patients $(3.9 \%)$ without L-thyroxine substitution moved to other treatment centres after a median diabetes duration of 7.5 years (1.2-19.5 years) and were lost to follow up for further analysis.

Of the 62 patients who had L-thyroxine treatment, 24 were boys $(39 \%)$ and 38 girls (61\%); their median age at commencement of treatment was 11.7 years ( $1-19$ years), and their median diabetes duration 3.4 years ( $0-12$ years). In three of these patients (one boy, two girls), hypothyroidism (increased TSH, decreased $\mathrm{T}_{3}$ and $\mathrm{T}_{4}$ ) was diagnosed 6, $\mathrm{l}$, and 56 months before diabetes onset (age at type 1 diabetes onset: 4.0, 11.2, and 12.5 years), respectively. At the start of L-thyroxine treatment, 59 of 62 patients (95\%) were positive for anti-TPO, and 53 (85\%) for both anti-TPO and anti-TG. Ultrasound abnormalities were present in all 62 patients requiring L-thyroxine treatment. Increased TSH levels (median $9.2 \mu \mathrm{U} / \mathrm{ml}, 4.7-100.0 \mu \mathrm{U} / \mathrm{ml}$ ) were present in 44 of those patients, and thyroid gland enlargement and typical ultrasound findings without TSH increase in 18 patients (TSH $3.2 \mu \mathrm{U} / \mathrm{ml}, 1.2-3.9 \mu \mathrm{U} / \mathrm{ml}$ ).

The cumulative incidence (SE) of AIT at the age of 18 years was $0.14(0.02)$ in the total group $(n=659)$, being significantly higher in female $(0.19(0.03), \mathrm{n}=301)$ than male (0.09 (0.02), $\mathrm{n}=358, \mathrm{p}=0.015$ ) patients (fig lA). At the beginning of puberty, after the age of 12 years, the AIT incidence in girls increased more than in boys (fig 1B). The cumulative incidence (SE) of AIT at 10 years of diabetes duration was $0.14(0.02)$ in the total group $(n=659)$ and was significantly higher in girls $(0.18(0.03), \mathrm{n}=301)$ than in boys (0.10 (0.02), $\mathrm{n}=358, \mathrm{p}=0.030$ ) (fig 2 ).

Forty five of 98 patients $(46 \%)$ with positive anti-TPO measurements during the study period required treatment with L-thyroxine. The cumulative incidence at 10 years of observation time was 0.69 (0.08) compared with $0.12(0.05)$ in 539 patients with negative anti-TPO measurements $(p<0.001)$ (fig $3 \mathrm{~A})$. Forty one of 92 patients $(45 \%)$ with positive anti-TG measurements developed AIT. The cumulative incidence at 10 years of observation time was $0.79(0.10)$
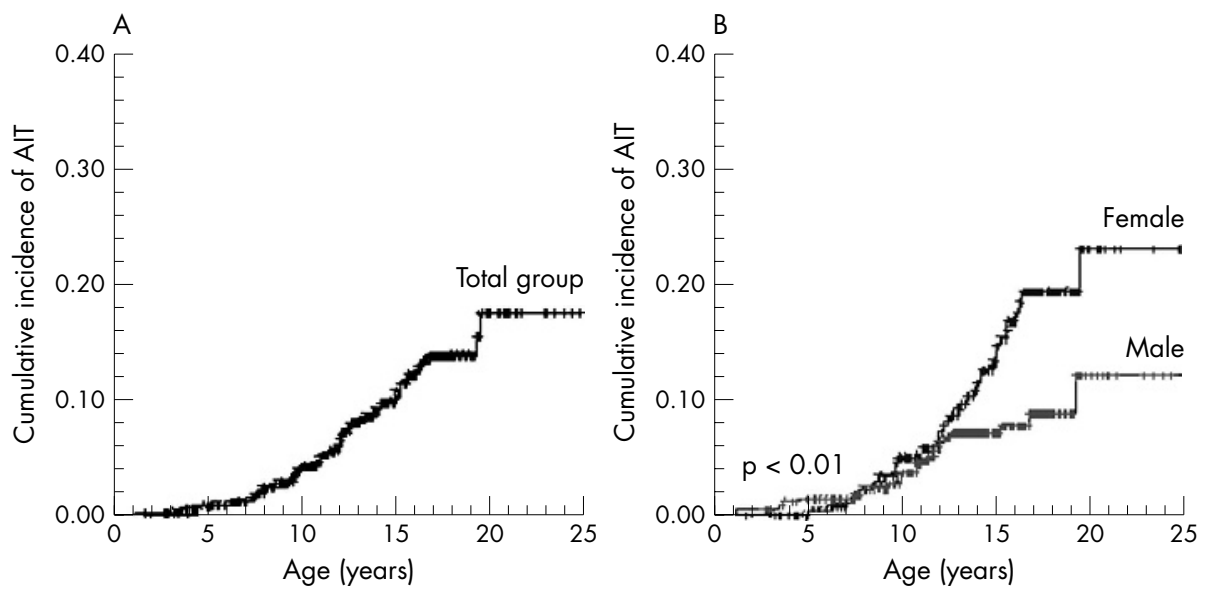

Figure 1 Cumulative incidence (probability) of autoimmune thyroiditis according to the age of 659 children and adolescents with type 1 diabetes: (A) in the total group, (B) stratified to gender of patients. 

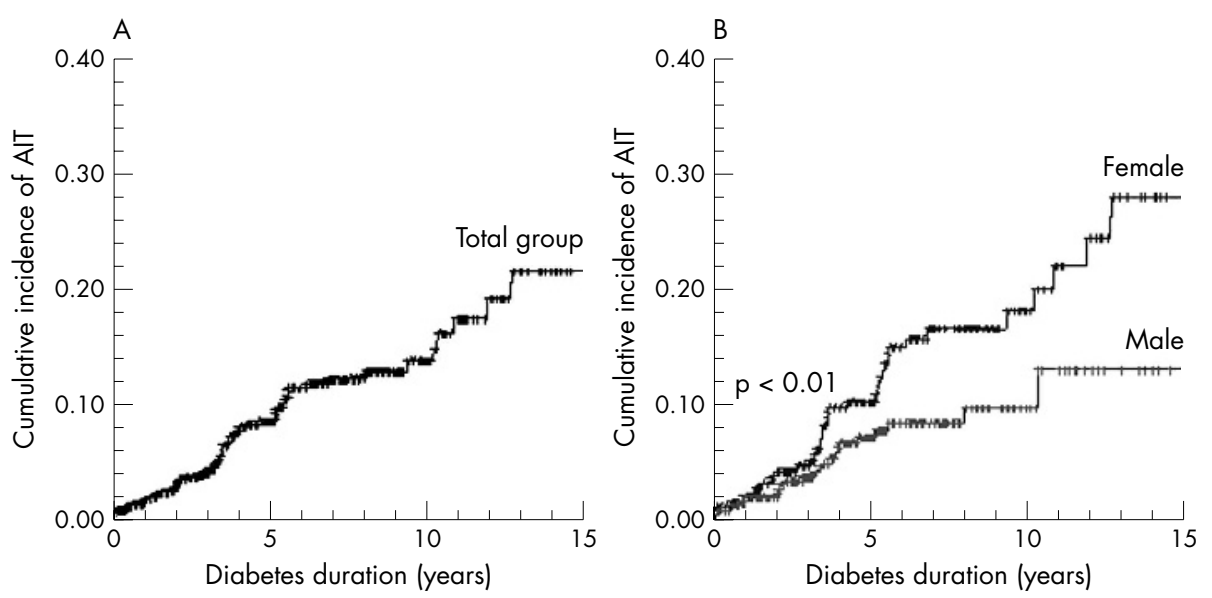

Figure 2 Cumulative incidence (probability) of autoimmune thyroiditis according to the duration of type 1 diabetes in 659 children and adolescents: (A) in the total group, (B) stratified to gender of patients.

compared with $0.12(0.04)$ in 545 patients with negative antiTG measurements ( $<<0.001)$ (fig 3B).

In the subgroup of 126 patients with repeated antibody measurements since type 1 diabetes onset, 23 patients (18\%) had positive thyroid antibody values already at type 1 diabetes onset: 19 patients with positive anti-TPO (15.1\%) and 19 patients with positive anti-TG (15.1\%). After three years of diabetes, a further three patients $(2.4 \%)$ became positive for both antibodies; after 5 years there was one patient $(0.8 \%)$ for anti-TPO and three patients $(2.4 \%)$ for anti-TG. All patients with significantly increased baseline values of anti-TPO $(\mathrm{n}=17,148-5340 \mathrm{U} / \mathrm{ml})$ and anti-TG $(\mathrm{n}=1 \mathrm{l}, 140-2000 \mathrm{U} / \mathrm{ml})$ at type 1 diabetes onset remained highly positive during the first five years of diabetes. In this subgroup of 30 patients with positive antibodies, 19 patients $(63 \%)$ required treatment with L-thyroxine.

\section{DISCUSSION}

These data clearly show that the incidence of autoimmune thyroiditis requiring treatment with L-thyroxine is highly increased in children and adolescents with type 1 diabetes compared to the general population. ${ }^{89}$ The cumulative incidence at 10 years of diabetes was found to be $14 \%$. In prior studies, the reported frequency of Hashimoto's thyroiditis was $3.5 \%$ (cross-sectional study) ${ }^{1}$ and $10 \%$ (mixed cross-sectional and longitudinal study), ${ }^{2}$ respectively. Signs of thyroid autoimmunity, such as the presence of thyroid autoantibodies, were apparent already at diabetes onset; however, thyroid dysfunction requiring treatment with L-thyroxine developed mostly thereafter. In our study, progression to clinical or subclinical hypothyroidism requiring treatment with L-thyroxine occurred during the first five years of diabetes in most patients with AIT, while no significant fluctuations of thyroid antibody titres were observed.

As in previous studies, ${ }^{10-13}$ females with type 1 diabetes were significantly predisposed to develop autoimmune thyroiditis. At the age of 18 years, almost every fifth girl with type 1 diabetes was diagnosed with an autoimmune thyroiditis requiring treatment with L-thyroxine. In the general population, girls are also prone to develop thyroid disease more than boys; ${ }^{3}$ however, it is noteworthy, that mostly female patients with type 1 diabetes are more likely to develop another autoimmune disease, like autoimmune thyroiditis, ${ }^{14}$ multiple sclerosis, ${ }^{15}$ and coeliac disease. ${ }^{16}{ }^{17}$ Given that type 1 diabetes is equally prevalent among males and females in most Caucasian populations or even a higher male-to-female ratio is shown in countries with a high diabetes incidence, ${ }^{18}$ this unequal gender distribution suggests that aetiological risk factors, unrelated to those for type 1 diabetes, may be associated with the simultaneous presence of more than one autoimmune disease.

In previous studies, we showed that the prevalence of thyroid antibodies in patients with type 1 diabetes increased
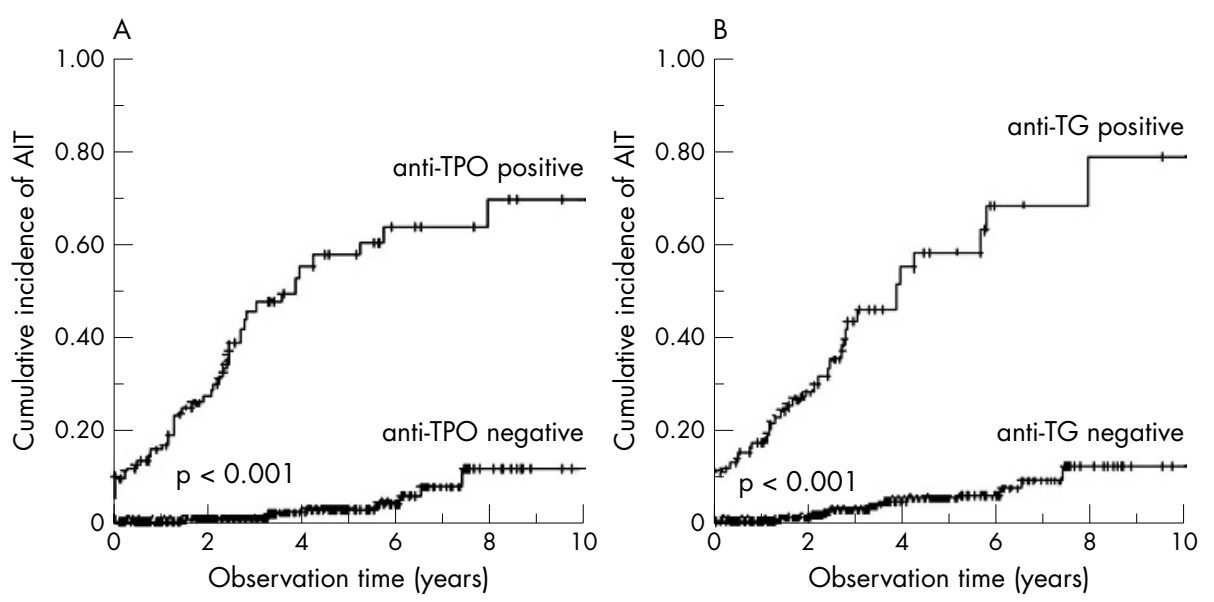

Figure 3 Cumulative incidence (probability) of autoimmune thyroiditis in 637 patients with type 1 diabetes according to the presence of antibodies against (A) thyroperoxidase (anti-TPO) and (B) thyroglobulin (anti-TG). 
with age. ${ }^{10}{ }^{12}$ In the present study, the same trend was found for the prevalence of thyroid dysfunction requiring treatment with L-thyroxine, while the duration of type 1 diabetes seemed to have no additionally independent influence on the development of clinical thyroiditis. Interestingly, in patients with type 1 diabetes and another autoimmune disease, diabetes onset mostly precedes the diagnosis of the other disorder. ${ }^{19}{ }^{20}$ Similarly, in the present study, except of three subjects, all patients developed clinically relevant thyroiditis after the manifestation of diabetes. The pathogenetic mechanism underlying the simultaneous occurrence of these clustered autoimmune diseases has not been clearly elucidated, even though there is evidence that common genetic determinants, in particular sharing of HLA risk alleles ${ }^{21-23}$ or other genes outside the HLA region (CTLA4 gene) ${ }^{24}{ }^{25}$ could be involved. Moreover, environmental factors are also assumed to be involved in the pathogenesis of these complex diseases. ${ }^{26}$ Whether the influence of exogenous agents leads to a faster onset of these autoimmune diseases, and particularly of type 1 diabetes, in patients with multiple diseases compared to those with only one disease needs to be assessed by epidemiological studies.

Despite the striking evidence that thyroid autoimmunity with subclinical hypothyroidism (increased TSH, normal $\mathrm{T}_{3}$ and $\mathrm{T}_{4}$, abnormal ultrasound images) is a frequent finding in children and adolescents with type 1 diabetes, there is still controversy concerning the necessity of therapeutic intervention in these patients. In a small observational study of 18 children and adolescents with autoimmune thyroiditis and subclinical hypothyroidism, ${ }^{27}$ seven patients were euthyroid, one patient became hypothyroid, and 10 patients continued to have increased TSH levels after an observation period of 47.3 months. However, in children with type 1 diabetes, there is strong evidence that hormonal thyroid abnormalities, even at a subclinical stage, may interfere with glycaemic metabolic control and increase insulin requirements. ${ }^{28}{ }^{29}$ Moreover, a significantly reduced growth rate was reported in young patients with diabetes and subclinical hypothyroidism with thyromegaly, particularly when TSH levels were higher than $10 \mathrm{mU} / \mathrm{l}$, while thyroid hormone replacement led to improved growth, especially in prepubertal patients. ${ }^{30}$ However, up to now, in children with subclinical hypothyroidism, both with and without type 1 diabetes, there has been a lack of longitudinal randomised study protocols, to examine the benefits of early treatment with L-thyroxine.

In conclusion, these data show that the development of autoimmune disease requiring treatment with L-thyroxine is significantly increased among young patients with type- 1 diabetes. Before the age of 18 years, approximately $14 \%$ of patients will require treatment for thyroid disease, while girls are more predisposed than boys. The first sign of these conditions may be the presence of positive thyroid autoantibodies, particularly anti-TPO, already at diabetes onset. The progression to TSH increase and/or ultrasound abnormalities mostly occurs within five years. However, development of thyroid autoimmunity may also occur during the further course of diabetes, particularly during puberty. Therefore, we would recommend that screening for thyroid disease should be performed at diabetes onset in all paediatric patients with type 1 diabetes. If the initial thyroid screening is positive, yearly laboratory (anti-TPO, anti-TG, TSH, $\mathrm{T}_{3}$, and $\mathrm{T}_{4}$ ) and ultrasound examinations are necessary in order to detect early thyroid dysfunction and initiate treatment with L-thyroxine. If the initial thyroid screening is negative, we recommend regular measurements of anti-TPO and TSH, even in the absence of clinical signs. Particularly from the age of 12 years or from the onset of puberty, TSH and antibody screening should be performed at yearly intervals.

\section{Authors' affiliations}

O Kordonouri, R Hartmann, D Deiss, M Wilms, A Grüters-Kieslich, Clinic of General Pediatrics, Otto-Heubner-Centrum, Charité, Campus Virchow-Klinikum, Humboldt University, Berlin, Germany

Competing interests: none declared

\section{REFERENCES}

1 Radetti G, Paganini C, Gentili L, et al. Frequency of Hashimoto's thyroiditis in children with type 1 diabetes mellitus. Acta Diabetol 1995;32:121-4.

2 Lorini R, d'Annunzio G, Vitali L, et al. IDDM and autoimmune thyroid disease in pediatric age group. J Pediatr Endocrinol Metab 1996:9(suppl 1):89-94.

3 Dayan CM, Daniels GH. Chronic autoimmune thyroiditis. N Engl J Med 1996;335:99-107.

4 Marcocci C, Vitti P, Cetani F, et al. Thyroid ultrasonography helps to identify patients with diffuse lymphocytic thyroiditis who are prone to develop hypothyroidism. J Clin Endocrinol Metab 1991;72:209-13.

5 ISPAD. Consensus guidelines for the management of type 1 diabetes mellitus in children and adolescents. Zeist, Netherlands: Medforum, 2000.

6 Liesenkötter KP, Kiebler A, Stach B, et al. Small thyroid volumes and normal iodine excretion in Berlin schoolchildren indicate full normalization of iodine supply. Exp Clin Endocrinol Diabetes 1997; 105(suppl 4):46-50.

7 Kabelitz M, Liesenkötter KP, Stach B, et al. The prevalence of anti-thyroid peroxidase antibodies and autoimmune thyroiditis in children and adolescents in an iodine replete area. Eur J Endocrinol 2003;148:301-7.

8 Tunbridge WM, Brewis $M$, French $J M$, et al. Natural history of autoimmune thyroiditis. BMJ (Clin Res Ed) 1981;282:258-62.

9 Vanderpump MP, Tunbridge WM. Epidemiology and prevention of clinical and subclinical hypothyroidism. Thyroid 2002; 12:839-47

10 Holl RW, Böhm B, Loos U, et al. Thyroid autoimmunity in children and adolescents with type 1 diabetes mellitus. Effect of age, gender and HLA type. Horm Res 1999;52:113-18.

11 Hansen D, Bennedbaek FN, Hansen LK, et al. Thyroid function, morphology and autoimmunity in young patients with insulin-dependent diabetes mellitus. Eur J Endocrinol 1999;140:512-18.

12 Kordonouri O, Klinghammer A, Lang EB, et al. Thyroid autoimmunity in children and adolescents with type 1 diabetes mellitus: a multicenter survey. Diabetes Care 2002;25:1346-50.

13 Kordonouri O, Deiss D, Danne T, et al. Predictivity of thyroid autoantibodies for the development of thyroid disorders in children and adolescents with type 1 diabetes. Diabet Med 2002;25:1346-50.

14 McCanlies E, O' Leary LA, Foley TP, et al. Hashimoto's thyroiditis and insulindependent diabetes mellitus: differences among individuals with and without abnormal thyroid function. J Clin Endocrinol Metab 1998;83:1548-51.

15 Dorman JS, Steenkiste AR, Burke JP, et al. Type 1 diabetes and multiple sclerosis: together at last. Diabetes Care 2003;26:3192-3.

16 Valerio G, Maiuri L, Troncone R, et al. Severe clinical onset of diabetes and increased prevalence of other autoimmune diseases in children with coeliac disease diagnosed before diabetes mellitus. Diabetologia 2002;45:1719-22.

17 Acerini CL, Ahmed ML, Ross KM, et al. Coeliac disease in children and adolescents with IDDM: clinical characteristics and response to gluten-free diet. Diabet Med 1998;15:38-44.

18 Karvonen M, Pitkaniemi M, Pitkaniemi J, et al. Sex difference in the incidence of insulin-dependent diabetes mellitus: an analysis of recent epidemiological data. Diabetes Metab Rev 1997;13:275-91.

19 Cronin CC, Shanahan F. Insulin-dependent diabetes mellitus and coeliac disease. Lancet 1997;349:1096-7.

20 Pocecco M, Ventura A. Coeliac disease and insulin-dependent diabetes mellitus: a causal association? Acta Paediatr 1995;84:1432-3.

21 Houlston RS, Tomlinson IP, Ford D, et al. Linkage analysis of candidate regions for coeliac disease genes. Hum Mol Genet 1997;6:1335-9.

22 Šumnik Z, Kolouškova S, Cinek O, et al. HLA-DQA 1*05-DQB1*0201 positivity predisposes to coeliac disease in $\mathrm{Czech}$ diabetic children. Acto Paediatr 2000;89:1426-30

23 Badenhoop K, Walfish PG, Rau H, et al. Susceptibility and resistance alleles of human leukocyte antigen (HLA) DQA1 and HLA DQB1 are shared in endocrine autoimmune diseases. J Clin Endocrinol Metab 1995:80:2112-17.

24 Naluai AT, Nilsson S, Samuelsson L, et al. The CTLA4/CD28 gene region on chromosome $2 q 33$ confers susceptibility to coeliac disease in a way possibly distinct from that of type 1 diabetes and other chronic inflammatory disorders. Tissue Antigens 2000;56:350-5.

25 Ueda $\mathbf{H}$, Howson JMM, Esposito L, et al. Association of the T-cell regulatory gene CTLA4 with susceptibility to autoimmune disease. Nature 2003;423:506-11

26 Leslie RD, Elliott RB. Early environmental events as a cause of IDDM: evidence and implications. Diabetes 1994;43:843-50.

27 Moore DC. Natural course of "subclinical" hypothyroidism in childhood and adolescence. Arch Pediatr Adolesc Med 1996;150:293-7.

28 Franzese A, Buono $P$, Mascolo $M$, et al. Thyroid autoimmunity starting during the course of type 1 diabetes denotes a subgroup of children with more severe diabetes. Diabetes Care 2000;23:1201-2.

29 Mohn A, Di Michele S, Di Luzio R, et al. The effect of subclinical hypothyroidism on metabolic control in children and adolescents with type 1 diabetes mellitus. Diabet Med 2002; 19:70-3.

30 Chase HP, Garg SK, Cockerham RS, et al. Thyroid hormone replacement and growth of children with subclinical hypothyroidism and diabetes. Diabet Med 1990;7:299-303. 